\title{
Towards abstract test methods for relay system testing
}

\author{
Jun $B i$ and Jianping Wu \\ Dept. of Computer Science, Tsinghua Univ., \\ Beijing, 100084, P.R.China \\ bj@csnet1.tsinghua.edu.cn \\ jinaping@cernet.edu.cn
}

\begin{abstract}
The traditional conformance testing theory and practice have been well used for testing the end system in the network. However, the relay system testing will play an important role in the computer network and distributed system. Abstract test method aims to enable test suite designers to use the most appropriate methods for their circumstances. This paper discusses the abstract test methods for relay system testing. At first, we introduce the model of the R-SUT (Relay System Under Test) and give the conceptual architecture of relay system testing. Then, several abstract test methods are proposed in this paper. At last, we illustrate some practical experience for testing the relay system, such as IP router, SMTP email server, and Packet Assemble/Disassembly (PAD), with the methods we present. These methods could be used for testing ATM switch too.
\end{abstract}

\section{Keywords}

Protocol conformance test, relay system, abstract test method 


\section{INTRODUCTION}

With the development of computer netowrks, lots of protocol software and hardware had been implemented by different manufacturers. At the same time, we have to spend more and more time to ensure the correctness of these different implementations. The aim of protocol conformance testing (PCT) is to verify the conformance between protocol implementation and its corresponding standard. Today, it is one of the most active fields on computer network and distributed system.

ISO/IEC 9646 [1] provides the OSI conformance testing methodology and framework. It had been widely used in the test practice for end system ([2][3][4][5][6][7] etc.). Notice that there are two kinds of system in the networks: end system and relay system. The traditional theory and practice of PCT usually focus on the end system testing, and the research for relay system testing is less. Today, relay is an important concept in TCP/IP, switched LAN, and high speed networks. The relay system, such as IP router, LAN switch, and ATM switch, played the important roles in these technologies [7]. Since the peer-to-peer and end-to-end model in ISO/OSI could not fit these relay technologies well, it is very important to study the test theory of relay systems.

Abstract test method aims to enable abstract test suite (ATS) designers to use the most appropriate method for their circumstances [8]. The testers test the behavior of implementation under test (IUT) by protocol data units (PDU) and abstract service primitives (ASP). In ISO/IEC 9646, there are some ripe abstract test methods for end system. These methods are based on ISO/OSI reference model. These test methods could be classified by point of control and observation (PCO), test coordination procedure and the position of tester. Because there is difference between the IUT of end system and the IUT of relay system, it is necessary to study the abstract test methods for relay system. Although there are two relay system test methods, "loop-back" (YL) and "transverse" (YT), defined in ISO 9646, their capabilities are limited. The YL test method is used for testing a relay system from only one subnetwork. Thus the disadvantage of this method is that the behaviour of the relay on only one side is directly observed [1]. The YT method has two PCOs, one on each subnetwork, and uses two test systems external from the IUT. So the procedures for coordinating the control applied to the two testers would be a big problem. To solve these problems and put the relay test into practice, we propose some new relay test methods. We hope it could help the test laboratory make the real test process continuously and high-efficiently.

This paper discusses the characteristics of relay system and presents abstract test methods for relay system testing. The rest of this paper is organized as follows. Section 2 analyzes the R-SUT model. A conceptual architecture of relay system testing is given in section 3 . In section 4 , several abstract test methods, RL, DL, LT, DT, CT and RT are proposed, then their characteristics 
are discussed in section 5. After a brief view of the protocol integrated test system (PITS) developed by Tsinghua University in section 6, we will introduce some practical experiences with relay system testing, such as the IP router, the SMTP mail relay, and the Packet Assemble/Disassembly (PAD), using the methods we present in section 7 . Finally, we give the conclusion.

\section{THE R-SUT MODEL}

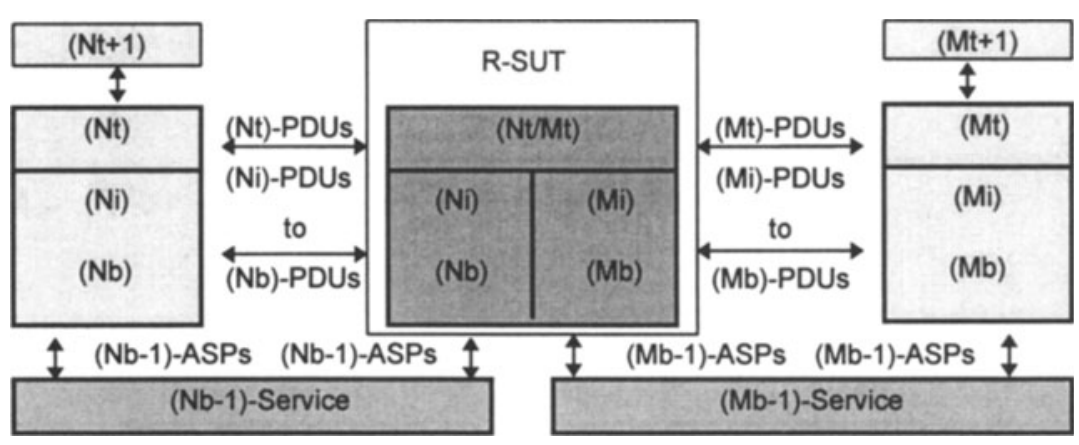

Figure 1 A model of the R-SUT.

There exists a relationship between the test methods and the configurations of the real network system to be tested [1]. There are two main configurations of system in a network:

(1) End system;

(2) Relay system.

Neither the term "relay system" nor "end system" has been defined by ISO nor other standard organizations, even though they are widely used in the field of data communication. The definition given by Cerf and Kirstein [9] is adopted here. It says that the collection of required hardware and software effect the interconnection of two or more data networks, enabling the passage of user data from one to another, is called a "relay system". This infers that a system connected only to one network will not be regarded as a relay system. All system other than relay system could be classified as end systems.

Now, we present a model of relay system under test (R-SUT) and it is shown in figure 1 . In this model, there are two protocol suites of subnetworks connected by the relay system. These two suites could be named " $N$ " and " $M$ ". If the two subnetworks have the same protocol architectures, $\mathbf{N}$ is equal to $\mathbf{M}$. The highest layer in the R-SUT is numbered "Nt" or "Mt" (for "top"), and the lowest is numbered "Nb" and "Mb" (for "bottom"). Notice that $\mathrm{Nt}$ is usually equal to Mt, and they realize the function of relay. For single-layer protocol R- 
SUTs, $\mathrm{Nt}$ (or Mt) is equal to $\mathrm{Nb}$ (or $\mathrm{Mb}$ ). In the following sections, the same notation will be used to refer to layers within the tester. The R-SUT may implement protocols in layers lower than " $\mathrm{Nb}$ ", but these are not of interest in the test method descriptions. For all test methods, ATSs specify test events at the lower tester PCO in terms of (Nb-1)-ASPs and (Mb-1)-ASPs and/or (Nt) to (Nb)-PDUs and (Mt) to (Mb)-PDUs. There are some features in R-SUTs:

(1) The relay layer is always the highest layer in a relay system. In another word, there is no upper layer above a relay function. So it is not necessary to control and observe its upper boundary by the (Nt+1)-ASPs and $(\mathrm{Mt}+1)$-ASPs.

(2) There are at least two subnetwork under a relay system, so the test events must be control and observed by the two sets of ASPs and PDUs.

\section{CONCEPTUAL ARCHITECTURE OF RELAY SYSTEM TESTING}

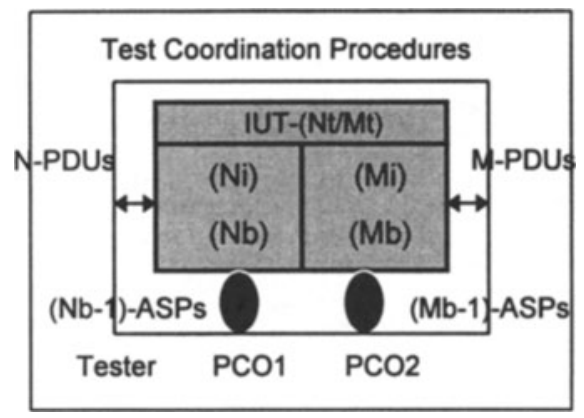

Figure 2 Conceptual architecture of relay system testing.

Abstract test methods are described in terms of what output from the IUT is observed and what inputs to it can be controlled. The starting point of developing abstract test methods is the conceptual testing architecture [1]. The conceptual architecture of the relay system testing is illustrated in figure 2 . It is a "blackbox" active testing architecture, based on the definition of behavior required by the IUT. The actions in this conceptual tester involve two sets of interactions: one for (N)-protocols and one for (M)-protocols. These can be controlled and observed at PCO1 and PCO2. Because of the ASPs above (Nt) is not specified, the tester is only lower tester (LT). LT would control and observe the (Nb-1)ASPs including (Nt) to (Nb)-PDUs at PCO1 and (Mb-1)-ASPs including (Mt) to (Mb)-PDUs at $\mathrm{PCO} 2$. 


\section{ABSTRACT TEST METHODS OF RELAY SYSTEM}

An abstract test method describes an abstract testing architecture consisting of testers and test coordination procedures, and their relationships to the test system and SUT. Each test method determines the PCOs and test events (i.e., ASPs and PDUs) which shall be used in an abstract test case for that test method. In this section, referring to the concepts and methods provided by ISO/IEC 9646, we propose 6 abstract test methods: RL, CL, LT, DT, CT, and RT. The ATSs should be specified in accordance with these methods.

\subsection{Remote loop-back test method (RL)}

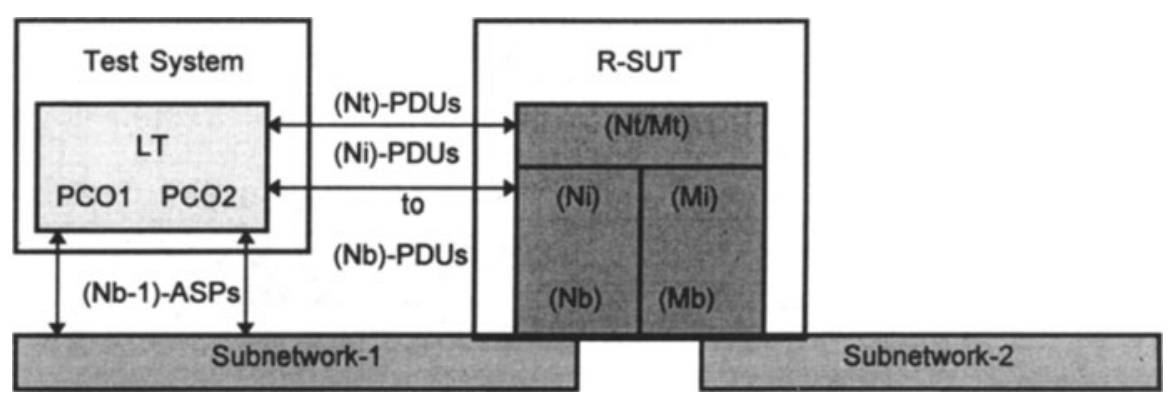

Figure 3 The RL test method.

The remote loop-back test method (RL) is illustrated in figure 3, just like the loopback method presented in ISO 9646. In this test method, there are two PCOs on one subnetwork at SAPs external from the (Nt)-Relay. For connectionoriented protocols, it requires that the two test connections are looped together on the far side of the relay system. This looping could be performed within the relay system or in the second subnetwork. For connectionless protocols, it requires that the PDUs are looped back within the second subnetwork and addressed to return the second PCO. This method enables a relay system to be tested without requiring test systems on two different subnetworks. Because there is only one lower tester (LT), the test coordination procedure of two PCOs would be very simple.

\subsection{Distributed loop-back test method (DL)}

The distributed loop-back test method (DL) is illustrated in figure 4. It uses a test responder (TR) in the extra destination host on the second subnetwork to send/ receive the PDUs to/from R-SUT. In test system, there are two PCOs for both side functions of R-SUT. When LT sends a PDU from PCO1 to the 
destination host, it would be relayed by R-SUT. TR located in the second subnetwork then controls and observes the events from R-SUT and returns it to LT through subsidiary test path (STP). This returned message could be obtained by LT from PCO2. The STP is also used for the test coordinating messages. In fact, this method combined the two lower testers (one should be in test system, and another in destination host) into one test system. Because the test suite including two PCOs is executed in one test system, the coordination of PCOs for both sides of R-SUT could be solved. It makes the test process automatically continuously and high-efficiently.

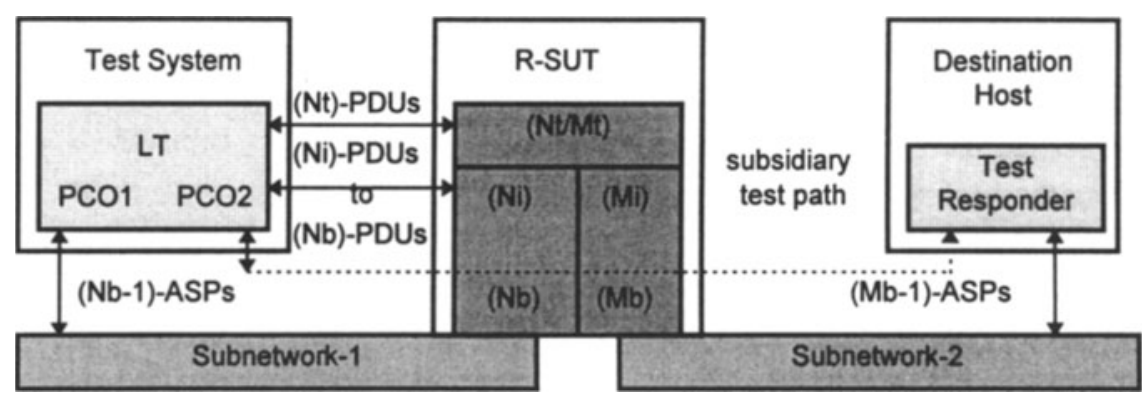

Figure 4 The DL test method.

\subsection{Local transverse test method (LT)}

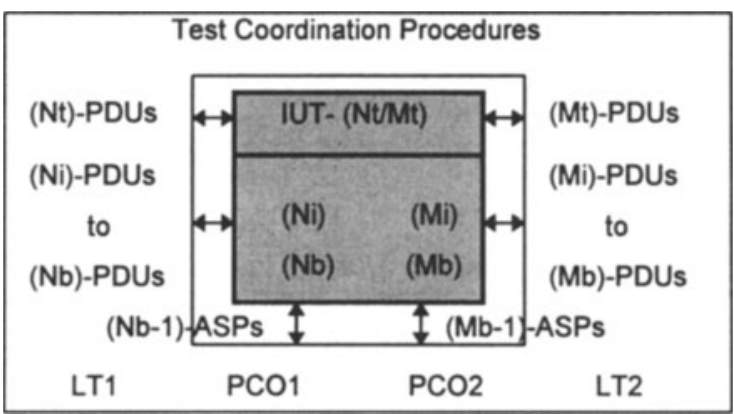

Figure 5 The LT test method.

Considering the transverse method presented in ISO 9646, we give the following four methods, LT, DT, CT and RT. The local transverse test method (LT) is illustrated in figure 5. This method have the following characteristics, 
(1) There is no upper tester.

(2) LT1, LT2, and SUT are in one local system, so the events occurred in SUT will be controlled and observed directly. However in many IUT, it seems not easy to find the required API for control and observation.

(3) The test events are specified by (Nb-1)-ASPs/(Nt), (Ni) to (Nb)-PDUs at PCO1 for LT1, and (Mb-1)-ASPs/(Mt), (Mi) to (Mb)-PDUs at PCO2 for LT2.

(4) Test coordination procedure between two lower testers may be realized in this local system by inter-process communication.

\subsection{Distributed transverse test method (DT)}

The distributed transverse test method (DT) is illustrated in figure 6. This method have the following characteristics,

(1) There is no upper tester.

(2) There are two lower testers, LT1 and LT2, in different test system. The events occurred in R-SUT will be controlled and observed on different subnetwork directly.

(3) The test events are specified by (Nb-1)-ASPs/(Nt), (Ni) to (Nb)-PDUs at PCO1 for LT1, and (Mb-1)-ASPs/(Mt), (Mi) to (Mb)-PDUs at PCO2 for LT2.

(4) Test coordination procedure between LT1 and LT2 would be realized by software or human, so it may be a problem for a real test system.

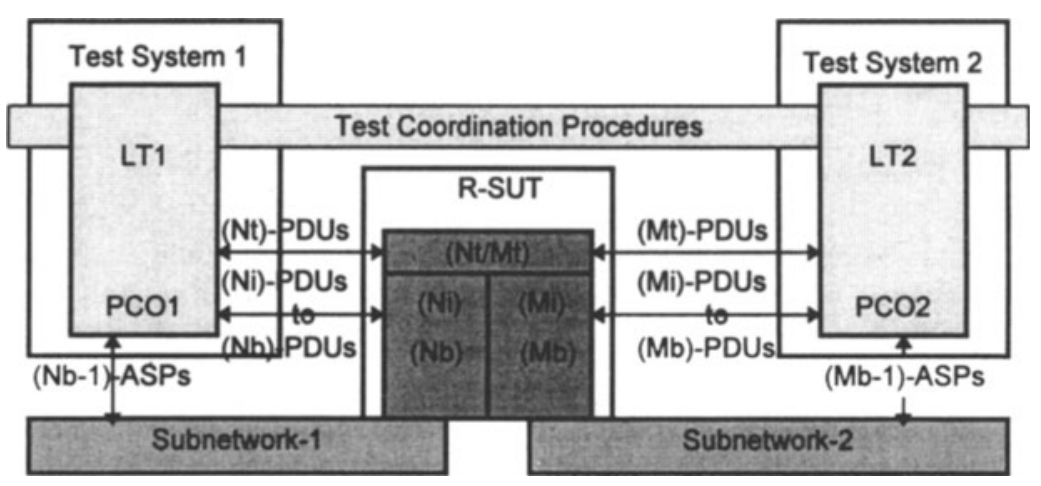

Figure 6 The DT test method.

\subsection{Coordinated transverse test method (CT)}

The coordination transverse test method (CT) is illustrated in figure 7. This method have the following characteristics, 
(1) There is no upper tester.

(2) Test coordinating procedures would be realized as a test management protocol (TMP) between LT1 and LT2, so it may be more difficult in practice.

(3) The test events are specified in terms of $(\mathrm{Nb}-1)-\mathrm{ASPs} /(\mathrm{Nt}),(\mathrm{Ni})$ to (Nb)-PDUs at PCO1 for LT1, and (Mb-1)-ASPs/(Mt), (Mi) to (Mb)-PDUs at $\mathrm{PCO} 2$ for LT2.

(4) LT1 and LT2 are in different test system, so the events occurred in RSUT will be controlled and observed on different subnetwork directly.

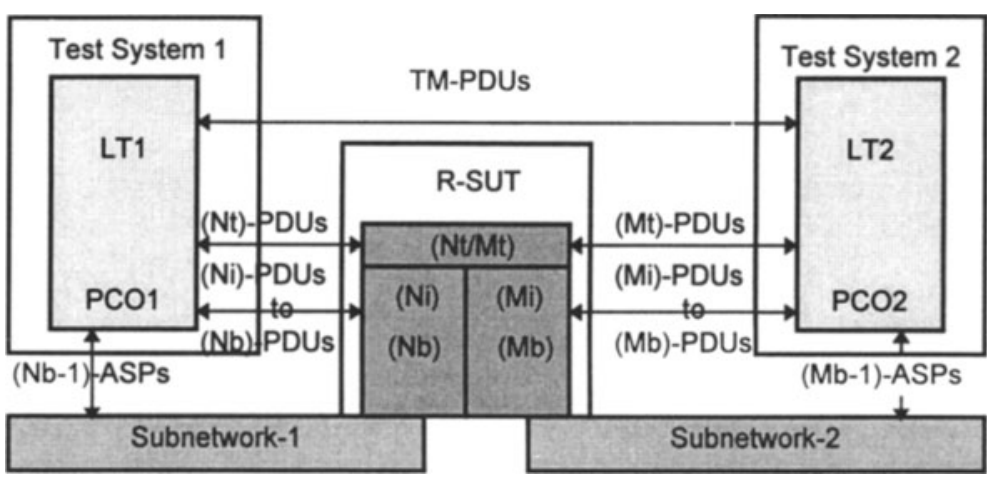

Figure 7 The CT test method.

\subsection{Remote transverse test method (RT)}

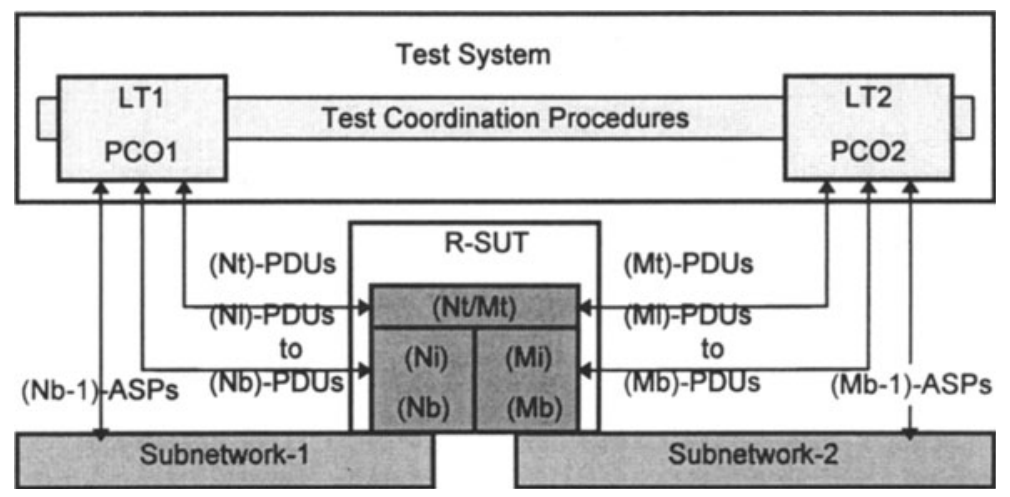

Figure 8 The RT test method.

The remote transverse test method (RT) is illustrated in figure 8. This method have the following characteristics, 
(1) There is no upper tester.

(2) LT1 and LT2 are in one test system and the events occurred in R-SUT will be controlled and observed on different subnetwork directly.

(3) The test events are specified in terms of $(\mathrm{Nb}-1)-\mathrm{ASPs} /(\mathrm{Nt})$, (Ni) to (Nb)-PDUs at PCO1 for LT1, and (Mb-1)-ASPs/(Mt), (Mi) to (Mb)-PDUs at PCO2 for LT2.

(4) Test coordination procedures between LT1 and LT2 would be realized in one test system as inter-process communication, so the test process would be highly efficient.

\section{COMPARISON OF THESE ABSTRACT TEST METHODS}

The abstract test methods we proposed could be generally divided into two kinds: loop-back method and transverse method.

The loop-back method is used for testing a relay system from one subnetwork. The advantage is that the procedures for coordinating the control applied to the two PCOs can be realized within a single test system. The disadvantage is that the relay behavior on only one side is directly observed. Thus, its behavior on the second subnetwork can not be properly assessed.

The transverse method is used for testing a relay system from two subnetworks. The advantages are:

(1) The behavior on each subnetwork could be controlled and observed.

(2) This method enables the relay system to be tested in the normal mode of operation.

The disadvantage is that the test coordination procedure may be much complex, because there are two LTs for different subnetworks. It is a big problem for the real test system designers.

In the methods of DT and CT, two LTs are located in two different test systems separately. So they could be used in the distributed test environment. In a real test system, the former is more simple but the test coordination procedure is more difficult. If the coordination could not be solved well, the test process would not be automatically and continuously. The later method could solve the coordination successfully. However, because of the implementation of TMP, there will raise more system cost.

In the methods of LT and RT, two LTs are located in one test system. So their test coordination procedures could be solved well. LT method could be used for a IUT which has clear interface. Because the tester and SUT are in the same system, its application would be limited. So, we think the realization of RT method has the following advantages:

(1) The two LTs are in one test system, so the common model and software could be used by these two testers when developing a real test system. It would deduce the system cost. 
(2) Test coordination procedures are simple and high-efficient. It could be realized as inter-process communication. It is better than TMP.

(3) The design of abstract test suite is simple. The designer only concerns the test event of two sides of R-SUT and need not pay attention to the coordination of the two sides.

(4) Use "black-box" testing and we need not the upper interface of IUT. So we need not add extra model in R-SUT. It could be used for different IUTs.

Moreover, the characteristics of these methods are shown in table 1.

Table 1 Characteristics of these test methods

\begin{tabular}{|l|c|c|c|c|c|c|}
\hline & \multicolumn{2}{|c|}{ Loop-back } & \multicolumn{4}{c|}{ Transverse } \\
\hline & RL & DL & LT & DT & CT & RT \\
\hline $\begin{array}{l}\text { Observation } \\
\text { of two } \\
\text { subnetwork }\end{array}$ & indirectly & directly & directly & directly & directly & directly \\
\hline Test systems & 1 & 1 & 1 & 2 & 2 & 1 \\
\hline $\begin{array}{l}\text { Test } \\
\text { coordination } \\
\text { procedures }\end{array}$ & $\begin{array}{l}\text { inter- } \\
\text { process, } \\
\text { simple, } \\
\text { automatic }\end{array}$ & $\begin{array}{l}\text { inter- } \\
\text { process, } \\
\text { simple, } \\
\text { automatic }\end{array}$ & $\begin{array}{l}\text { inter- } \\
\text { process, } \\
\text { simple, } \\
\text { automatic, }\end{array}$ & $\begin{array}{l}\text { by human, } \\
\text { and } \\
\text { may be } \\
\text { single step }\end{array}$ & $\begin{array}{l}\text { using } \\
\text { TMP, } \\
\text { complex, } \\
\text { automatic }\end{array}$ & $\begin{array}{l}\text { inter- } \\
\text { process, } \\
\text { simple, } \\
\text { automatic }\end{array}$ \\
\hline $\begin{array}{l}\text { Need } \\
\text { extra TR }\end{array}$ & no & yes & no & no & no & no \\
\hline $\begin{array}{l}\text { IUT } \\
\text { independence }\end{array}$ & yes & yes & no & yes & yes & yes \\
\hline
\end{tabular}

\section{PROTOCOL INTEGRATED TESTING SYSTEM (PITS)}

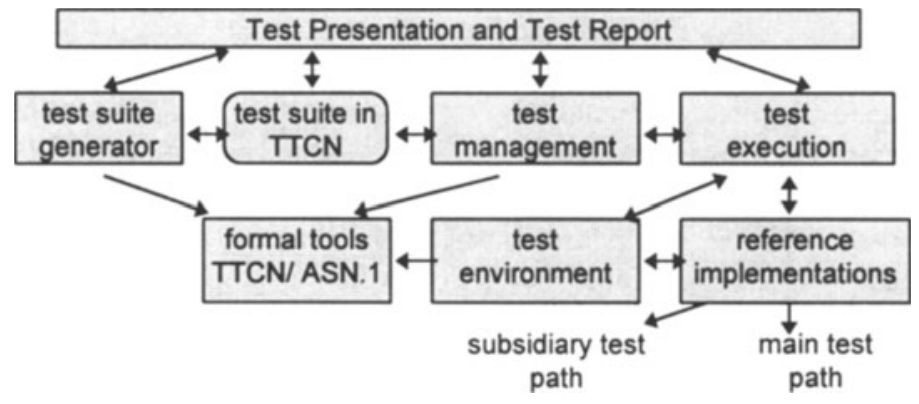

Figure 9 The protocol integrated testing system PITS.

In this section, we will introduce the protocol integrated test system PITS. The 
PITS aims to provide a basic platform to test different protocols by different test methods. It could be used for both the conformance testing, the interoperability testing, and the performance testing. It has been used for testing many implementations of end systems and relay systems. The PITS shown in figure 9, is composed of the following main components: test presentation and test report, test management [10], test execution [11], test suite generator, reference implementations, formal support tools and test software environment. The TTCN test suite is generated from EBE specification, which could be translated from LOTOS and Estelle specification [12]. The Reference Implementation (RI) is a very important part in this test system. It is the special protocol implementation and acts as the lower communicating support for controlling and observing the events occurred in test execution (TE).

The following objectives guided our design and implementation effort:

(1) Accordance with ISO protocol testing standards. All the ideas, methods and terminology adopted in our PITS strictly follow ISO 9646 protocol testing standard. In our PITS, all the protocol reference implementations and the services accord with corresponding ISO standards. The test suite (TS) is formally described in TTCN, which is defined in ISO 9646-3 [1].

(2) Flexibility and independence. In our TTCN based TE, test suite is executed according to the operational semantics of TTCN, so this method is flexible and independent on the protocol being tested. It could be regard as a general executing mechanism for any TS in TTCN. So it can test all the protocols whose TS is in TTCN. Then any new protocol can be tested by our PITS, only with fulfillment of its TS in TTCN.

(3) Efficient test execution. The parallel interpreting improves the test executing efficiency. When a test case is being interpreted, the most possible next test case is being interpreted. TTCN based TE interprets a test case just before its execution. It will allow testing operator with the more possibilities to control the testing process, such as single step testing and supervising.

\section{PRACTICAL EXPERIENCE WITH RELAY SYSTEM TESTING}

In this section, we will introduce some practical testing experiences .with the relay test methods using PITS.

\subsection{Testing IP router}

Today, the IP router is one of the most important relay system in Internet. The function or purpose of IP is to move datagrams through an interconnected set of networks. This is done by passing the datagrams from one Internet module to another until the destination is reached. The IP modules reside in hosts and routers in the Internet. The datagrams are routed from one IP module to another 
through individual networks basing on the interpretation of the Internet address. Thus, one important mechanism of the Internet protocol is the IP addressing. In the routing from one IP module to another, datagrams may need to traverse a network whose maximum packet size is smaller than the size of the datagram. To overcome this difficulty, a fragmentation mechanism is provided in the IP protocol. Errors detected may be reported via the Internet Control Message Protocol (ICMP).

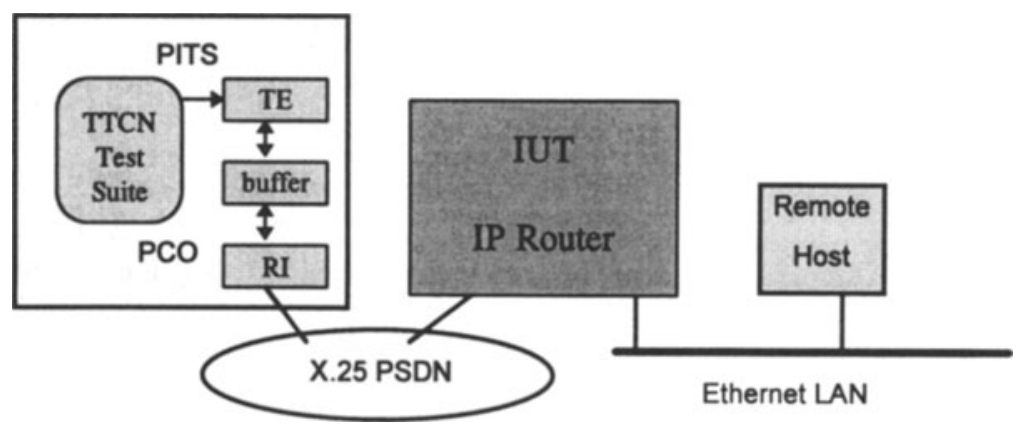

Figure 10 Testing IP router with DL.

We use PITS to test IP router with RL method. It is shown in figure 10. This IP router connects two subnetworks: Ethernet LAN and X.25 public data network. When PITS sends a IP/ICMP datagram (for example ECHO) to the remote host, after routing and addressing, it will be forward by IP router from X.25 PSDN to Ethernet LAN. The response IP datagram could address to PITS and be observed at PCO. We have designed a TTCN based test suite for IP router. This test suite contains 32 test cases and the following is an example. The test purpose is shown in this test case. Now the test suite is only a prototype for verifying the new test architecture. We are developing the complete IP test suite. Then we could test IP from more subnetwork and test more IP options.

Table 2 A test case of IP routing

\begin{tabular}{|c|c|c|c|c|c|}
\hline \multicolumn{6}{|c|}{ Test Case Dynamic Behavior } \\
\hline \multicolumn{6}{|c|}{ 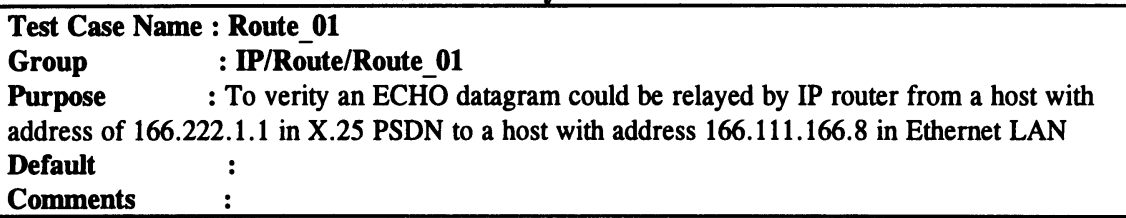 } \\
\hline $\mathbf{N r}$ & Label & Behavior Description & Constraint Reference & Verdict & Comments \\
\hline 1 & & ! Echoreq & Echoreq_01 & & (1) \\
\hline
\end{tabular}




\begin{tabular}{|c|c|c|c|c|}
\hline $\begin{array}{l}2 \\
3 \\
4 \\
5 \\
6 \\
7\end{array}$ & $\begin{array}{l}\text { Start T01 } \\
\qquad \begin{array}{l}\text { ? Echorsp } \\
\text { ? Otherwise } \\
\text { ? Timeout T01 }\end{array}\end{array}$ & Echorsp_01 & $\begin{array}{l}\text { PASS } \\
\text { FAIL } \\
\text { FAIL }\end{array}$ & (2) \\
\hline $\begin{array}{l}\text { Det } \\
\text { (1) } \\
\text { (2) }\end{array}$ & $\begin{array}{l}1.1, \mathrm{DA}=166.111 .16 \\
66.8, \mathrm{DA}=166.222\end{array}$ & & & \\
\hline
\end{tabular}

\subsection{Testing PAD}

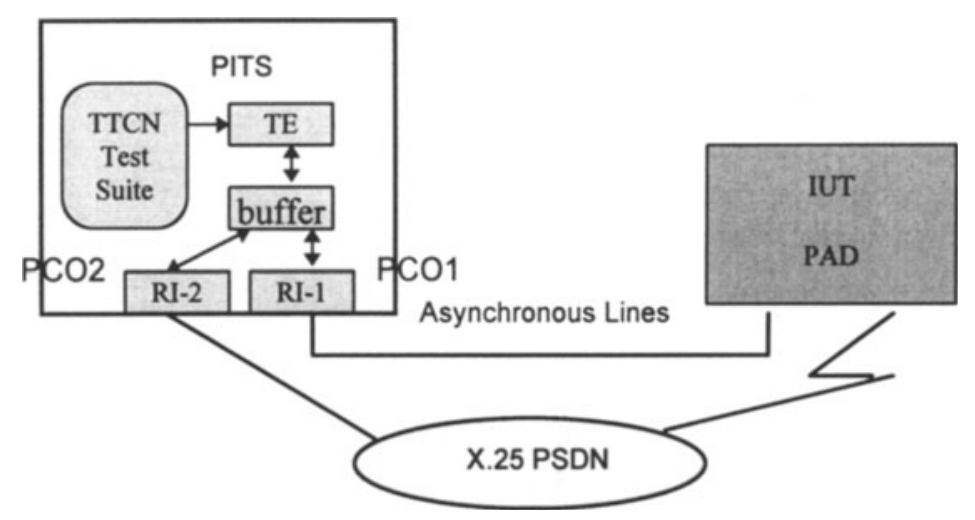

Figure 11 Testing PAD with RT.

CCITT defined three recommendations (X.3/X.28/X.29) about packet assembly/disassembly device (PAD) in public network. Recommendation X.3 defines a set of parameters for PAD. X.29 defines the procedures between a PAD and a packet mode DTE or another PAD, and X.28 defines the DTE/DCE interface for a start-stop mode DTE accessing PAD. The PAD is a special relay system. One side of a PAD is the X.25 public data network for packet mode DTE, and another side is asynchronous lines for terminals.

We use RT method to test PAD. There are two PCOs in the test suite. So we implement two RIs to control and observe the test event in/out the IUT. When TE interpreting and executing the TTCN based test suite, the test events would be send to the corresponding RI from the buffer according to their PCOs. Figure 11 shows using PITS to test the relay function of PAD. Because of RT's advantages, we think this architecture is a good approach to test switch equipment in LAN and WAN.

The TTCN based PAD test suite we designed contains 234 test cases. The 
following is an example. The parameter 1 allows the start-stop DTE to initiate an escape from the "data transfer" state or the "connection in progress" state in order to send PAD command signals. Value 0 of parameter 1 indicates that recall is impossible; value 1 indicates that recall using a character DLE; value 32 to 126 using graphic character defined by user. In this test case, we verify the function of value 1. Pre_9 is a preamble and CHK_9 is a verification sequence for state 9 .

Table 3 A test case of PAD

\begin{tabular}{|c|c|c|c|c|c|}
\hline \multicolumn{6}{|c|}{ Test Case Dynamic Behavior } \\
\hline \multicolumn{2}{|c|}{$\begin{array}{l}\text { Test Case Na } \\
\text { Group } \\
\text { Purpose } \\
\text { impossible. } \\
\text { Default } \\
\text { Comments } \\
\end{array}$} & $\begin{array}{l}\text { : } \mathbf{S P} \mathbf{1 0 1} \\
\text { : PAD/SP/ } \\
\text { : Verity that with parameter } 11 \\
: \\
:\end{array}$ & set to $0, \mathrm{PAD}$ & 11 using a c & racter is \\
\hline$\overline{\mathbf{N r}}$ & Label & Behavior Description & $\begin{array}{l}\text { Constraint } \\
\text { Reference }\end{array}$ & Verdict & Comments \\
\hline $\begin{array}{l}1 \\
2 \\
3 \\
4 \\
4 \\
5 \\
6 \\
7 \\
8 \\
8\end{array}$ & & $\begin{array}{l}+ \text { Pre }^{9} \\
\text { PCO2 ! N_DT_req (MSET) } \\
\text { PCO1! DLE } \\
\text { PCO2! N_DT_req (DT) } \\
\text { Start TM01 } \\
\text { PCO1? CHAR } \\
\text { + CHK 9 } \\
\text { PCO1? Otherwise } \\
\text { PCO1? Timeout TM01 }\end{array}$ & $\begin{array}{l}\text { MSET_20 } \\
\text { DLE_01 } \\
\text { DT_11 } \\
\text { CHAR_01 }\end{array}$ & $\begin{array}{l}\text { (PASS) } \\
\text { FAIL } \\
\text { FAIL }\end{array}$ & $\begin{array}{l}\text { set } 1,0 \\
\text { 'A' } \\
\text { 'A' }\end{array}$ \\
\hline
\end{tabular}

\subsection{Testing SMTP relay server}

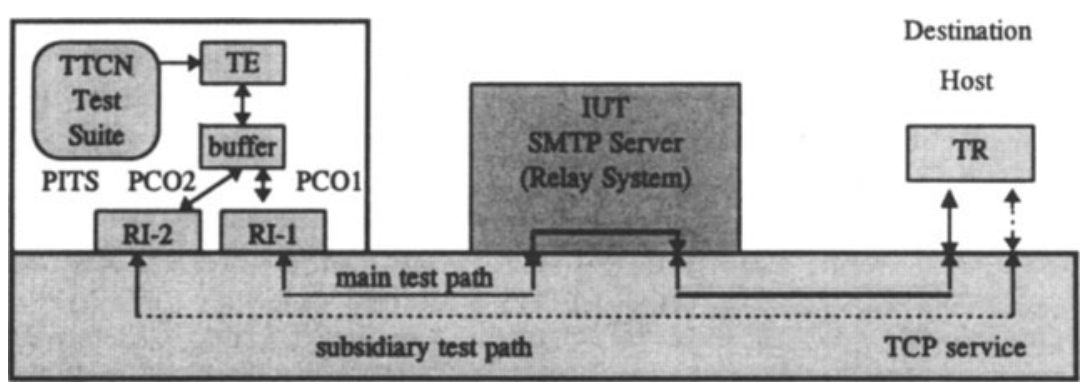

Figure 12 Test architecture of relaying email. 
SMTP is designed in the RFC standards. RFC 821 specifies the definition of SMTP and RFC 822 specifies the syntax of test message that sent as email with BNF (Backus-Naur Form). The objective of SMTP is to transfer email reliably and efficiently. SMTP is independent of the particular transmission subsystem and TCP is the most popular transmission subsystem. An important feature of SMTP is its capability to relay mail across transport service environments. A transport service provides an interprocess communication environment (IPCE). Emails can be communicated between processes in different IPCEs by relaying through a process connected to two (or more) IPCEs. More specifically, mail can be relayed between hosts on different transport systems by a server on both transport systems.

We use the DL method to test the relay function of SMTP mail server. Figure 12 shows this testing architecture. There are two PCOs for both side functions of IUT. The TTCN test suite contains 89 test cases in total. There is an example in [13].

\section{CONCLUSION}

In ISO 9646, there are some standard abstract test methods for the end system and two methods for the relay system. For testing a real relay system, these methods are too simple to direct the test activities well. We have proposed six abstract test methods (RL, DL, LT, DT, CT, and RT) for relay system testing. They are the recommendations for real test system. The characteristics of these test methods had been discussed in section 5 . These test methods would be selected according to their characteristic and the situation of SUT. We had implemented three test methods (RL, DL, RT) in PITS using the Sun Sparc workstation and Solaris 2.4. It had been presented to be very successful in the testing of IP router, SMTP mail server, and PAD. Now we are focusing on the other three test methods (LT, DT, CT) in the testing of ATM switch, and the more complex relay system such as the Internet routing protocols. We believe that relay system will be more important in the future, especially for Internet and high speed network. We hope there come more efforts for relay system tesing using the test methods proposed in this paper.

\section{REFERENCES}

[1] ISO, Information Processing System - Open System Interconnection - OSI Conformance Testing Methodology and Framework, ISO/IEC 9646, 1991.

[2] K. Katsuyama and F. Sato, Strategic testing environment with formal description techniques, IEEE Trans. on Computer, Vol. 40, No.4, 1991.

[3] S.T.Chanson, et al., The UBC Protocol Testing Environment, in O.Rafiq, 
editors, 6th IFIP IWPTS, 1993.

[4] C.S.Lu, et al., An Implementation of CMIP/CMISE conformance testing system, in T.Mizuno, et. al., editors, 7th IFIP IWPTS, 1994.

[5] K.Y.Kim, et al., Experiences with the design of B-ISDN integrated test system (BITS), in A.Cavalli, et. al., editors, 8th IFIP IWPTS, 1995.

[6] W.Geyer, S.Hesse and N.Newrly, TSE-P - a highly flexible tool for testing network management applications using simulation, in B.Baumgarten, et. al., editors, 9th IFIP IWTCS, 1996.

[7] T.W.Kim, et. al., Field trial and quality test of ATM switching system in Korea, in B.Baumgarten, et. al., editors, 9th IFIP IWTCS, 1996.

[8] D.Rayner, OSI conformance testing, Computer network \& ISDN systems, Vol.14, 1987.

[9] V.G.Cerf and R.T.Kirstein, Issues in packet-network interconnection, proceeding of IEEE, Vol.66, No.11, 1978.

[10] J. Tian and J. Wu, Test management and TTCN based test sequencing, in A.Cavalli, et. al., editors, 8th IFIP IWPTS, 1995.

[11] Y. Wang, J. Wu and R. Hao, An approach to TTCN-based test execution, in T.Mizuno, et. al., editors, 7th IFIP IWPTS, 1994.

[12] J. Wu and S.T.Chanson, Translation from LOTOS and Estelle specifications to extended transition system and its verification, in 2nd IFIP FORTE, 1989.

[13] J.Bi and J.Wu, et.al., Application of a TTCN based conformance test environment on the Internet email protocol, to appear in 10th IFIP IWTCS, 1997. 